\title{
Avaliação de Métodos de Classificação de Glaucoma em Imagens do Fundo do Olho
}

\author{
Débora Ferreira de Assis * Pedro Motta* \\ Paulo César Cortez* Fábio Cisne Ribeiro* \\ * Universide Federal do Ceará \\ e-mail:\{debora.ferreira, pedro.motta, cortez, fabio\}@lesc.ufc.br.
}

\begin{abstract}
Glaucoma is an asymptomatic ocular disease at the onset which, if left untreated, can lead to blindness. The World Health Organization (WHO) has estimated that by 2020 glaucoma should affect 80 million people and by 2040 will be 111.5 million. In this context, the present work aims to compare methods of automatic classification of glaucoma to aid in its diagnosis by medical specialists. For this, two models are developed based on the extraction of characteristics of eye fundus images obtained from the public base RIM-ONE v2, and also uses a simple convolutional neural network (CNN). In the first model, texture characteristics, histogram information and image colors are extracted, which are submitted to the Principal Component Analysis (PCA) to evaluate nine classical classifiers including Multilayers perceptron (MLP) . In the evaluation are used Accuracy, sensitivity, specificity and area under curve (AUC) . The results demonstrate that the best performance is obtained with the MLP classifier with PCA use in the characteristic descriptors, with accuracy of $90.11 \%$, sensitivity $97.44 \%$, specificity of $84.62 \%$, and AUC equal to 0.90 .

Resumo: O glaucoma é uma doença ocular assintomática no início que, se não for tratada a tempo, pode levar a cegueira. A Organização Mundial de Saúde (OMS) estimou que em 2020 o glaucoma deve afetar 80 milhões de pessoas e em 2040 serão 111,5 milhões. Neste contexto, o presente trabalho visa comparar métodos de classificação automática do glaucoma para auxiliar no seu diagnóstico por médicos especialistas. Para tanto, são desenvolvidos dois modelos com base na extração de características de imagens de fundo de olho, obtidas da base pública RIMONE v2, e também se utiliza de uma rede neural convolucional (CNN) simples. No primeiro modelo, extraem-se características de textura, informações de histogramas e cores das imagens, que são submetidas à Análise de Componentes Principais (PCA) para, em seguida, realizar a avaliação de nove classificadores clássicos, incluindo o Multilayers perceptron (MLP). No segundo modelo, adicionam-se características oculares geométricas extraídas: Cup to Disk Ratio (CDR), Neuro-Retinal Rim (NRR) e Blood Vessel Ratio (BVR). Empregam-se na avaliação a acurácia, sensibilidade, especificidade e área sob a curva (area under curve - AUC). Os resultados demonstram que o melhor desempenho é obtido com o classificador MLP com uso do PCA nos descritores de características, com acurácia de 90,11\%, sensibilidade 97,44\%, especificidade de $84,62 \%$, e AUC igual a 0,90 .
\end{abstract}

Keywords: Glaucoma; Convolutional Neural Network; MLP; PCA; Segmentation of the Optic Nerve.

Palavras-chaves: Glaucoma; Classificação; Rede Neural Convolucional; MLP; PCA; Segmentação do Nervo Óptico.

\section{INTRODUÇÃO}

O glaucoma consiste em um conjunto de doenças neuropáticas oculares que danificam o nervo óptico, geralmente é dividido em dois tipos: de ângulo aberto e de ângulo fechado. O glaucoma de ângulo aberto se desenvolve lentamente e tem sintomas que são percebidos somente em estágios avançados da doença. Já o glaucoma de ângulo fechado tem sintomas mais imediatos e destrutivos, podendo causar perda total de visão em poucos dias (MANTRAVADI; VADHAR, 2015).

\footnotetext{
* Agradecimentos à CAPES - Código de Financiamento 001.

${ }^{\star}$ Trabalho suportado pelo projeto Proc. $311436 / 2015-3$ CNPq.
}

O exame mais indicado, por sua eficiência e custo, para o diagnóstico de glaucoma é a fundoscopia que é uma técnica de observação do fundo do olho (retina e outras estruturas internas). No entanto, sua análise é limitada pela visão humana que incorpora fatores subjetivos, tornandoa dependente do especialista. Devido a isso é crescente os estudos para identificação de glaucoma, a partir de imagens de fundo do olho geradas por um retinógrafo.

Informações divulgadas na BBC indicaram que segundo as projeções da Organização Mundial de Saúde (OMS), estima-se que o glaucoma deve afetar 80 milhões de pessoas em 2020, e esse número tende a crescer em 2040, alcançando 111,5 milhões de pessoas. Por consequência, 
pesquisas que auxiliem no diagnóstico de glaucoma possuem grande importância para a saúde pública (THAM et al., 2014; JONAS et al., 2017).

O desenvolvimento de sistemas computacionais que automatizam o processo de detecção e diagnóstico de doenças oftalmológicas, tem ganhado cada vez mais importância para dar suporte à análise estruturas oculares, auxiliando especialistas na detecção precoce de alterações patológicas (SILVA, 2018). Existem duas abordagens para classificação com base em imagens: aprendizado de máquina e métodos de aprendizado profundo. O primeiro método consiste em extrair características da imagem usando descritores e depois, utiliza-se essas características como entrada para um algoritmo classificador; e o segundo método extraem características, analisam padrões e classificam em um único processo (LECUN; BENGIO; HINTON, 2015).

Recentemente, diversos trabalhos foram desenvolvidos para a detecção de glaucoma a partir de imagens de fundo do olho. Septiarini et. al. (2018) utilizaram aprendizado por máquinas por meio do classificador k-NN, e GómezValverde et.al. (2019) desenvolveram técnicas de aprendizado profundo através de Redes Neurais Convolucionais (CNN) para identificação de glaucoma com base neste tipo de imagens. Apesar do bom desempenho, esses métodos não realizam uma avaliação de técnicas de classificação.

Neste contexto, este trabalho apresenta uma metodologia para a avaliação de métodos de classificação para identificar o glaucoma a partir de imagens do fundo de olho. Esta metodologia utiliza a aprendizagem de máquinas, na qual são comparados dois modelos de extração de características para a avaliação de desempenho dos classificadores. Também é avaliado um método de aprendizado profundo baseado em uma CNN simples.

\section{LEVANTAMENTO BIBLIOGRÁFICO}

$\mathrm{Na}$ literatura pesquisada, alguns trabalhos têm obtidos resultados considerados eficientes na identificação de glaucoma. Recentes trabalhos têm se destacado na utilização de descritores de características aplicadas a classificadores, bem como técnicas baseadas em aprendizado profundo.

Acharya et. al. (2015) extraíram dos coeficientes da transformada de Gabor características como média, variância, assimetria, curtose, energia, entropias de Shannon e Rényi. Essas variáveis foram submetidas à análise de componentes principais (PCA) para reduzir a dimensionalidade, as 23 componentes resultantes são aplicadas no classificador SVM. Isto produziu uma precisão média de $93,10 \%$, sensibilidade de $89,75 \%$ e especificidade de $96,20 \%$.

Em Samanta et. al. (2015) extraíram características de Haralick e aplicaram a uma rede neural de propagação reversa $(\mathrm{BPN})$, resultando numa classificação para o glaucoma com uma precisão de $96 \%$.

O estudo realizado por Balasubramanian et. al. (2016) fornece uma avaliação da detecção de glaucoma usando a extração do Recurso Histograma de Gradientes Orientados (HOG) juntamente à classificação SVM da imagem de fundo do olho com a extração de vasos sanguíneos, usando o filtro Gabor. A precisão obtida é de $83,3 \%$ e a sensibilidade de $75,0 \%$.
No trabalho de Araújo (2017) são extraídas características dos índices de diversidade de Shannon e McIntrosh como descritores de textura e se utiliza SVM para a classificação. O método produz uma acurácia média de $88,35 \%$, sensibilidade média de $84,50 \%$ e especificidade média de $91,37 \%$.

Septiarini (2018) extraiu de imagens do nervo óptico a média, suavidade e terceiro momento. Em seguida, usase a seleção de variáveis através da correlação e para a classificação é aplicado o algoritmo de k-NN, o qual alcança uma precisão de $95,24 \%$.

No estudo realizado por Zahoo et. al. (2018), o disco óptico foi segmentado e pré-processado para destacar a área da neuroretinal rim (NRR) e a escavação. Um perceptron multicamada com um vetor de característica $12-\mathrm{D}$ é usado para segmentação da escavação. Um classificador baseado em árvore de decisão é empregado para identificar as imagens glaucomatosas e não glaucomatosas. Este método produz uma acurácia 95,30\%, sensibilidade de 96,31\% e especificidade de $95,33 \%$.

Gómez-Valverde et.al. (2019) exploraram o uso de aprendizado de transferência versus arquiteturas recém-definidas. A base utilizada é uma junção da ESPERANZA, RIMONE (v1, v2 e v3) e DRISHTI-GS. O melhor desempenho alcançado foi utilizando aprendizagem por transferência com o modelo VGG19 produzindo uma AUC de 0,94, acurácia de $88,05 \%$, sensibilidade de $87,01 \%$ e especificidade de $89,01 \%$.

\section{METODOLOGIA}

As imagens utilizadas são do banco de dados RIM-ONE v2, esse é um dataset público disponível com imagens no formato JPG, no sistema RGB, de fundo do olho. Esta base de dados possui 455 imagens, sendo 255 de pacientes saudáveis e $200 \mathrm{com}$ glaucoma. As imagens são redimensionadas para iguais dimensões $200 \times 200$. Na figura 1 estão expostas duas imagens típicas: (a) paciente saudável, (b) paciente com glaucoma.

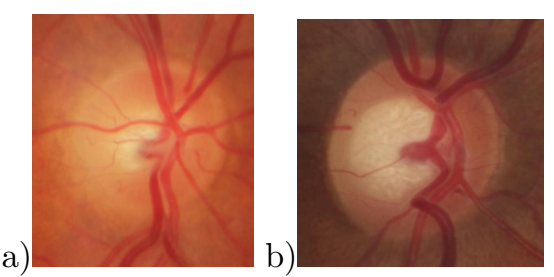

Figura 1. Imagens típicas do conjunto de dados RIM_ONE v2 (a) pacientes saudáveis, e (b) pacientes com glaucoma.

Desenvolvem-se dois modelos baseados na extração de características de imagens de fundo de olho. No modelo 1 , são extraídas características das imagens com os seguintes descritores: Local Binary Pattern (LPB), Histogram of Oriented Gradients (HOG), entropia de Shannon, textura de Haralick, momentos de Hu e informações de cores do histograma. O LBP, o HOG e a Haralick são descritores de textura. A entropia de Shannon é calculada para detectar variações sutis na distribuição do nível de cinza local. Já o HOG é utilizado para obter informações da distribuição de cores das imagens. 


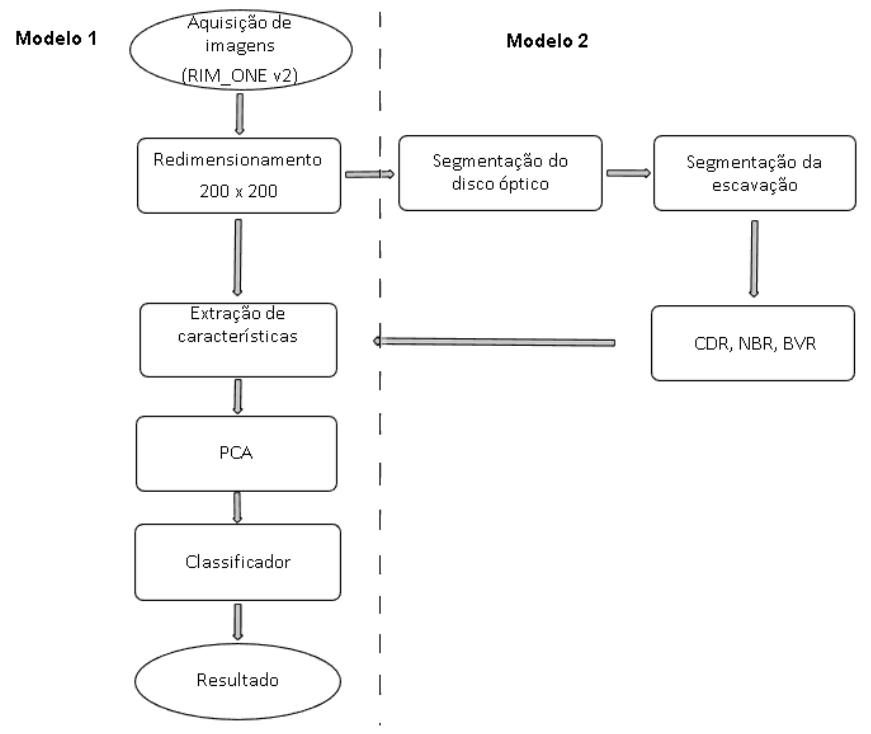

Figura 2. Fluxograma dos modelos.

No modelo 2, as características oculares geométricas são adicionadas aos descritores do modelo 1. Esses parâmetros calculados são Cup to Disk Ratio (CDR), Neuro-Retinal Rim (NRR) e Blood Vessel Ratio (BVR), que consideram o tamanho da escavação, o deslocamento dos vasos e a regra ISNT (Inferior, Superior, Nasal, Temporal), mostrados na Figura 3. Essas características, quando irregulares, são indicativos de glaucoma (ABRÀMOFF; GARVIN; SONKA, 2010).

\subsection{Segmentação}

A segmentação do disco óptico consiste por duas etapas: remoção dos vasos com a operação morfológica top hat e uma filtragem mediana. Em seguida, o Método de Contorno Ativo (MCA) é utilizado para encontrar as bordas do disco óptico. Esta é uma técnica de segmentação de objetos em imagens digitais que localiza bordas do objeto, utilizando-se de informações da imagem (KASS; WITKIN; TERZOPOULOS, 1988).

Para segmentar a escavação, o componente verde $G_{i+1}$ da imagem é recalculado por $G_{i+1}=G_{i}-\left(\mu_{i}+\sigma_{i}\right)$, em que $G_{i}$ componente verde original $\mu_{i}$ é a média da imagem e $\sigma_{i}$ o seu desvio padrão. Em seguida, aplica-se a Limiarização de Otsu para encontrar os valores na imagem que pertencem à escavação. Observa-se nas Figura 4.a, 4.b e 4.c o resultado da segmentação do disco óptico, da escavação e dos vasos extraídos, respectivamente.

$\mathrm{Na}$ figura 3.b, é possível analisar a divisão ISNT da área do disco óptico em que, para um disco saudável tem-se a seguinte ordem para a área dos quadrantes:

Inferior $(\mathrm{I})>\operatorname{Superior}(\mathrm{S})>\operatorname{Nasal}(\mathrm{N})>$ Temporal $(\mathrm{T})$.

O CDR é a razão entre a área da escavação e a área do disco óptico dado por (KAUSU, et al., 2018)

$$
C D R=\frac{\text { Área da escavação }}{\text { Área do disco óptico }},
$$

olhos com CDR maiores que 0,3 normalmente são glaucomatosos.
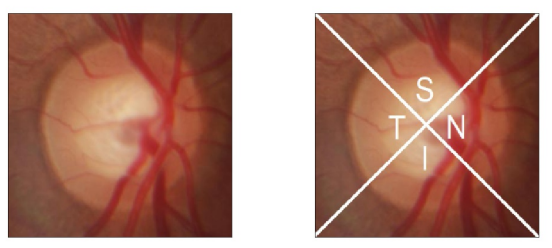

Figura 3. a) Fundoscopia. b) Quadrantes da fundoscopia ISNT.

Para calcular o NRR, remove-se a escavação de dentro do disco óptico e divide-se a área restante em quadrantes. Em seguida, encontra-se a razão entre a área dos quadrantes verticais e horizontais dada por (KAUSU, et al., 2018)

$$
N R R=\frac{\mathrm{I}+\mathrm{S}}{\mathrm{N}+\mathrm{T}} .
$$

Olhos glaucomatosos geralmente têm NRR menor que um.

Se um olho é saudável, seus vasos sanguíneos concentramse nos quadrantes superior e inferior. Calculando a razão entre os vasos sanguíneos dos quadrantes verticais e horizontais, encontra-se o Blood Vessel Ratio (BVR) (KAUSU, et al., 2018)

$$
B V R=\frac{\operatorname{Vasos} \mathrm{I}+\operatorname{Vasos} \mathrm{S}}{\operatorname{Vasos} \mathrm{N}+\operatorname{Vasos} \mathrm{T}} .
$$

Em geral, olhos glaucomatosos possuem BVR menor que um. Na Figura 4.c pode-se observar os vasos extraídos para o cálculo do BVR.

a)

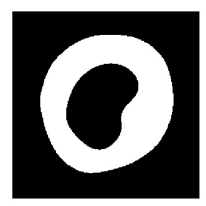

b)

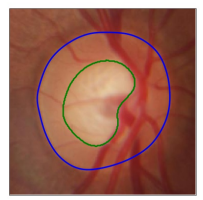

c)

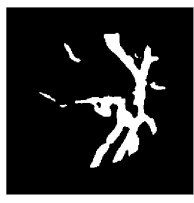

Figura 4. (a) Rima Neural. (b) Imagem com o contorno do DO e da escavação. (c) Vasos sanguíneos dentro do DO.

\subsection{Classificação}

A partir das características extraídas das imagens, são avaliados diferentes classificadores para detecção automática de glaucoma: Regressão Logística (RL), Análise Linear Discriminante (LDA), $K$-nearest neigbor (k-NN) com $k=3$, Árvores de Decisão (CART), Random Forest (RF), Gradient Boosted Decision Trees (GBDT), Naive Bayes Gaussiano (NB), Support Vector Machine (SVM) e Multilayers perceptron (MLP).

A regressão logística é comumente aplicada à classificação binária, utiliza-se a sigmóide como função de ativação. O LDA é uma técnica estatística multivariada para discriminar e classificar objetos, considerando probabilidades a priori. Por outro lado, o k-NN é a técnica mais simples de aprendizado de máquinas.

As árvores de decisão são modelos simples e eficazes para problemas de classificação e de regressão, são rápidas de treinar e fáceis de interpretar. Já o RF é um modelo 
que combina os resultados de diversas árvores de decisão geradas independentemente que melhora previsão geral. Por outro lado, as GBDT funcionam de forma semelhante ao $\mathrm{RF}$ e é composta por várias árvores e, diferentemente do $\mathrm{RF}$, cada árvore de decisão é cultivada de forma a melhorar os erros das árvores anteriores.

O NB é um classificador probabilístico baseado no teorema de Bayes, que desconsidera a correlação entre as variáveis. Os SVM são considerados grandes classificadores que tentam separar os dados usando uma grande margem, neste trabalho é utilizado o kernel linear.

O MLP é composto de vários neurônios ligados entre si por sinapses com pesos, a função de ativação utilizada é a Unidade Linear Retificada (ReLU), sendo treinada por métodos de retopropagação e gradiente descendente.

Com intuito de aprimorar os resultados, utiliza-se a PCA para reduzir a dimensionalidade das características extraídas. Esta técnica transforma ortogonalmente as coordenadas originais de um conjunto de dados em um novo conjunto de coordenadas. Neste trabalho, utilizam-se as componentes que quando somadas representam 95\% da variância explicada.

A rede neural convolucional $(\mathrm{CNN})$ é treinada a partir do zero, é uma rede composta por um conjunto simples com 3 camadas de convolução, uma ativação de ReLU e seguida por camadas de MaxPooling. O dropout foi utilizado por ajudar a reduzir overfitting, impedindo que uma camada veja duas vezes o mesmo padrão. Além disso, são colocadas duas camadas totalmente conectadas. O modelo finaliza com uma única unidade e uma ativação sigmóide. Para treinar o modelo foi utilizado a função de perda binarycrossentropy. As imagens de entrada para a CNN foram convertidas para o tamanho $256 \times 256$. A arquitetura da rede é descrita na Tabela 1.

Tabela 1. Arquitetura da $\mathrm{CNN}$

\begin{tabular}{lccc}
\hline Processo & Parâmetro & Tamanho & Ativação \\
\hline Convolução & 32 & $255 \times 255$ & Relu \\
MaxPooling & 32 & $127 \times 127$ & Relu \\
Convolução & 32 & $126 \times 126$ & Relu \\
MaxPooling & 32 & $63 \times 63$ & Relu \\
Convolução & 64 & $62 \times 62$ & Relu \\
MaxPooling & 64 & $31 \times 31$ & Relu \\
Dropout & 64 & $31 \times 31$ & Relu \\
Flatten & 46656 & - & Relu \\
Densa & 64 & - & Relu \\
Dropout & 64 & - & Relu \\
Densa & 1 & - & Sigmóide \\
\hline
\end{tabular}

\subsection{Métricas de Avaliação}

As métricas para comparação do desempenho dos classificadores são: acurácia, sensibilidade, especificidade e AUC. Antes da definição é importante entender alguns conceitos básicos.
Tabela 2. Matriz de confusão - Validade de um teste de diagnóstico

\begin{tabular}{lcc}
\hline \multirow{2}{*}{ Teste } & \multicolumn{2}{c}{ Doença (padrão ouro) } \\
\cline { 2 - 3 } & Presente & Ausente \\
\hline Positivo & VP & FP \\
Negativo & FN & VN \\
\hline
\end{tabular}

A acurácia (A) indica a proporção de acerto, ou seja, se o teste classificou corretamente os indivíduos doentes e sadios. A sensibilidade $(\mathrm{S})$ é a capacidade do teste diagnósticar corretamente os doentes. A especificidade (E) é a capacidade do teste diagnosticar corretamente os indivíduos saudáveis.

$$
A=\frac{V P+V N}{n}, \quad S=\frac{V P}{V P+F N}, \quad E=\frac{V N}{V N+F P},
$$

em que VP é verdadeiro positivo, VN é verdadeiro negativo, FP é falso positivo, FN é falsos negativo, e n é a quantidade total de observações.

A Curva ROC é uma métrica de verificação de qualidade do modelo, que determina a probabilidade de classificar um indivíduo com glaucoma (sensibilidade), contra a probabilidade de classificar incorretamente este indivíduo com glaucoma (1-especificidade). A qualidade do modelo é obtida através da área sob a curva (area under curve AUC), que fornece uma medida de taxa de acerto. Portanto, quanto mais distante da linha diagonal melhor será o modelo (ROBIN, 2015).

\section{RESULTADOS E DISCUSSÕES}

O conjunto de dados foi dividido em treinamento e teste, $80 \%$ e $20 \%$, respectivamente, com 384 imagens para treino e 91 para teste.

Considerando todas as características do modelo 1, o melhor desempenho deu-se com o random forest alcançando $A=85,71 \%, S=92,31 \%, E=80,77 \%$, e AUC 0,86 . Seguido pelo classificador MLP com $A=85,71 \%, S=$ $88,37 \%, E=83,33 \%$ e AUC 0,86.

Tabela 4. Resultado dos classificadores do modelo $1+$ PCA.

\begin{tabular}{ccccc}
\hline Classificador & Acurácia & Sensibilidade & Especificidade & AUC \\
\hline RL & $85,71 \%$ & $88,37 \%$ & $83,33 \%$ & 0,86 \\
LDA & $84,62 \%$ & $90,00 \%$ & $80,39 \%$ & 0,85 \\
KNN & $82,42 \%$ & $87,50 \%$ & $78,43 \%$ & 0,82 \\
CART & $63,74 \%$ & $69,70 \%$ & $60,34 \%$ & 0,64 \\
RF & $67,03 \%$ & $100,00 \%$ & $60,00 \%$ & 0,67 \\
XGB & $80,22 \%$ & $88,89 \%$ & $74,55 \%$ & 0,80 \\
NB & $57,14 \%$ & $88,89 \%$ & $53,66 \%$ & 0,58 \\
SVM & $84,62 \%$ & $88,10 \%$ & $81,63 \%$ & 0,85 \\
MLP & $90,11 \%$ & $97,44 \%$ & $84,62 \%$ & 0,90 \\
\hline
\end{tabular}

Com a aplicação da PCA para redução de dimensionalidade, houve uma discreta melhora na taxa de acerto dos classificadores XGB, SVM e MLP. Entretanto, os classificadores RF e NB apresentam uma queda quanto à assertividade na classificação, a taxa de acurácia reduziu consideravelmente $67,03 \%$ e $57,14 \%$, respectivamente. O classificador com melhor desempenho dentre todos foi o 
Tabela 3. Comparação de desempenho do método proposto com outros métodos.

\begin{tabular}{lllllllll}
\hline Autores & Dados & Qtd & Tr & Te & Método & A (\%) & S (\%) & E (\%) \\
\hline Acharya et. al. (2015) & Privada & 510 & 459 & 51 & SVM & 93,10 & 89,75 & 96,20 \\
Samantha et. al. (2015) & RIM-ONE v2 & 455 & 422 & 23 & BPN & 96,16 & 99,51 & 90,44 \\
Balasubramanian et. al. (2016) & - & 30 & 24 & 6 & SVM & 83,30 & 75,00 & - \\
Araújo et. al. (2017) & RIM-ONE v2 & 455 & 384 & 91 & SVM & 88,35 & 84,50 & 91,37 \\
Septiarini et. al. (2018) & Privada & 84 & 42 & 42 & K-NN & 95,24 & - & - \\
Zahoo et. al. (2018) & RIM-ONE+HRF & 205 & - & - & CART & 95,30 & 96,31 & 95,33 \\
Gómez-Valverde (2019) & & 2313 & 1734 & 579 & VGG19 & 88,05 & 87,01 & 89,01 \\
Método proposto & RIM-ONE v2 & 455 & 384 & 91 & MLP+PCA & 90,11 & 97,44 & 84,62 \\
Método proposto & RIM-ONE v2 & 455 & 384 & 91 & CNN & 87,91 & 85,71 & 91,87 \\
\hline
\end{tabular}

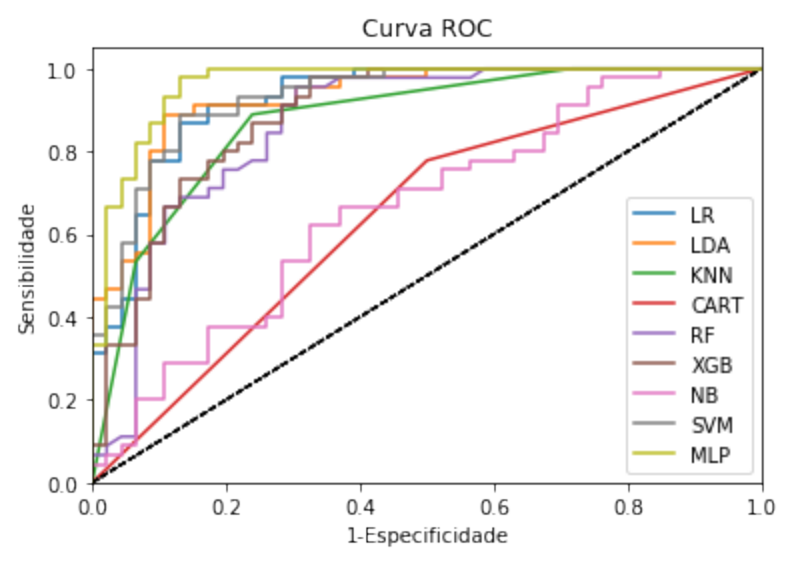

Figura 5. Curva ROC para cada classificador do modelo 1 + PCA.

MLP, com taxa de acerto de 90,11\%, sensibilidade $97,44 \%$, especificidade $84,62 \%$, e AUC 0,90 . A Tabela 4 mostra o resultado dos classificadores com uso do PCA nas características extraídas, e a Figura 5 mostra o gráfico da curva ROC referente a cada um desses classificadores.

Considerando o modelo 2, os resultados com a aplicação do PCA apresentaram melhor desempenho do que os resultados sem a utilização do mesmo, apesar disso, os resultados ainda foram inferiores em comparação ao modelo 1. Isso indica que o método de segmentação precisa ser melhorado, em especial a segmentação da escavação, pois ao invés de auxiliar na identificação do glaucoma, as características geométricas obtidas confundiram os classificadores, levando-os a respostas errôneas.

Os resultados que incluíam as características geométricas apresentam taxas com menores valores AUC. O MLP ainda foi o melhor classificador, com valores de acurácia, sensibilidade, especificidade e AUC iguais a $83,52 \%$, $86,00 \%, 80,49 \%$ e 0,83 , respectivamente.

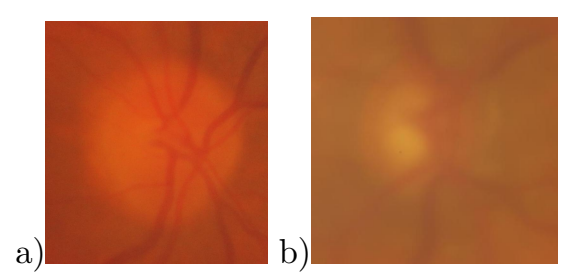

Figura 6. Classificação errônea das imagens, (a) falso positivo e (b) falso negativo.

A Figura 6 mostra imagens do fundo de olho das classificações errôneas. A Figura 6.a é um falso positivo, ou seja, o teste indica que o paciente tem glaucoma quando na verdade ele é saudável; e a Figura 6.b é um falso negativo, o teste indica que o paciente está saudável quando na verdade tem glaucoma.

A qualidade dessas imagens prejudica o classificador na separação de classes, ocorrendo assim o erro no momento de identificar a qual pertence. Algumas características são responsáveis pela classificação errada das classes, como: imagens bastante borradas, intensidades de cores próximas e dificuldade de distinção entre disco e escavação. Desse modo, quanto melhor a qualidade e o ângulo da imagem mais eficiente será a extração de características relevantes, como bordas e contornos, para especificar diferentes imagens.

Para a CNN, o conjunto de dados foi dividido em treino, validação e teste, $90 \%, 10 \%$ e $10 \%$, respectivamente. O treinamento é realizado com 100 épocas. Os resultados obtidos alcançaram uma taxa de acurácia média de 87,91\%, sensibilidade $85,71 \%$, especificidade $91,87 \%$ e AUC igual a 0,87 . Este resultado não foi tão bom quanto o MLP, e o custo computacional é significativamente maior. Entretanto, com o aprimoramento da rede e/ou o aumento da quantidade de dados, espera-se que os resultados possam ser melhores.

A Tabela 3 mostra os resultados e informações relevantes dos trabalhos avaliados em comparação com os modelos propostos. As informações são referentes aos: autores, base de dados utilizada, quantidade de imagens (Qtd), quantidade de treino ( $\mathrm{Tr}$ ), quantidade de teste (Te), método de classificação, e as métricas acurácia (\% A), sensibilidade (\% S) e especificidade (\% E).

O classificador SVM é o mais utilizado dentre os trabalhos avaliados, alcançou resultados com desempenho entre $83 \%$ e $93 \%$, entretanto com uma quantidade reduzida no conjunto treino, comprometendo o desempenho destes métodos. Os modelos propostos neste trabalho obtiveram resultados na faixa média dos trabalhos avaliados, apesar de não ter alcançado os melhores valores, o desempenho da classificação mostrou ser promissor e com baixo custo computacional. Neste sentido, pode ser uma técnica relevante para o auxílio do diagnóstico médico na detecção do glaucoma, em especial, por ter sido testado (Te) com uma quantidade maior de imagens do que os demais métodos que resultaram em acurácias maiores. Ressaltando ainda que a quantidade de dados para teste é igual somente em Araújo et. al. (2017), no qual apresenta a acurácia de $88,35 \%$, inferior ao resultado obtido neste trabalho. 
A CNN apresentou resultados relevantes com acurácia de $87,91 \%$, comparado com o trabalho de Gómez-Valverde (2019) é visto que os resultados foram bastante próximos. Este resultado pode ser melhorado com o desenvolvimento de um modelo mais robusto, apesar disso o custo computacional é alto em relação aos outros classificadores, em especial o MLP+PCA que mostrou melhor desempenho dentre as técnicas avaliadas.

\section{CONCLUSÕES}

Em suma, foram extraídas características das imagens e aplicadas em diversos classificadores com a finalidade de verificar qual apresenta o melhor desempenho para identificação automática do glaucoma. Os resultados obtidos mostraram uma eficiência quanto ao uso do PCA para redução de dimensionalidade e o MLP para classificar a imagem de acordo com sua classe, alcançando uma acurácia de $90,11 \%$. O custo computacional é baixo e as classificações são realizadas de forma rápida. Apesar dos resultados não apresentarem uma acurácia tão alta quanto alguns trabalhos mencionados, as taxas médias obtidas mostraram ser satisfatórias.

\section{TRABALHOS FUTUROS}

Para trabalhos futuros é considerado melhorar a segmentação da escavação a fim de obter resultados mais precisos para as características oculares geométricas. Utilizar aprendizagem por transferência para extração de características das imagens e aplicar nos classificadores estudados anteriormente. Além disso, aprimorar a rede convolucional para obter melhor desempenho no conjunto teste. Para aumentar o conjunto de dados é considerado empregar outros formatos de imagens públicas, para isso recorrer a detecção automática através do LBP, e segmentar somente a região do disco óptico.

\section{AGRADECIMENTOS}

O presente trabalho foi realizado com apoio da Coordenação de Aperfeiçoamento de Pessoal de Nível Superior Brasil (CAPES) - Código de Financiamento 001. E com apoio do Laboratório de Engenharia de Sistemas de Computação (LESC) - UFC.

\section{REFERÊNCIAS}

[1] Abràmoff, M. D., Garvin, M. K., \& Sonka, M. (2010). Retinal imaging and image analysis. IEEE reviews in biomedical engineering, 3, 169-208.

[2] Acharya, U. R., Ng, E. Y. K., Eugene, L. W. J., Noronha, K. P., Min, L. C., Nayak, K. P., \& Bhandary, S. V. (2015). Decision support system for the glaucoma using Gabor transformation. Biomedical Signal Processing and Control, 15, 18-26.

[3] Araújo, J. D. L., de Paiva, A. C., de Almeida, J. D., Neto, O. P. S., de Sousa, J. A., Silva, A. C., \& Júnior, G. B. (2017, July). Diagnóstico de glaucoma em imagens de fundo de olho utilizando os Índices de diversidade de shannon e mcintosh. In $17^{\circ}$ Workshop de Informática Médica (WIM 2017) (Vol. 17, No. 1/2017). SBC.
[4] Balasubramanian, T., Krishnan, S., Mohanakrishnan, M., Rao, K. R., Kumar, C. V., \& Nirmala, K. (2016, December). HOG feature based SVM classification of glaucomatous fundus image with extraction of blood vessels. In 2016 IEEE Annual India Conference (INDICON) (pp. 1-4). IEEE.

[5] Gómez-Valverde, J. J., Antón, A., Fatti, G., Liefers, B., Herranz, A., Santos, A., ... \& Ledesma-Carbayo, M. J. (2019). Automatic glaucoma classification using color fundus images based on convolutional neural networks and transfer learning. Biomedical optics express, 10(2), 892-913.

[6] Gonzalez, R. C., \& Woods, R. C. (2010). Processamento digital de imagens .3. ed. São. Paulo: Pearson / Prentice Hall.

[7] https://www.bbc.com/portuguese/geral-47979880.

[8] Jonas, J. B. et al. (2017). Glaucoma. The Lancet. Volume 390, Issue 10108, Pages 2183-2193, ISSN 0140-6736.

[9] Kass, M., Witkin, A. \& Terzopoulos, D. (1988). Snakes: Active Contour Models . International Journal of Computer Vision, v. 1, n. 4,p.321-331,1988.

[10] Kausu, T. R., et al., (2018). Combation of clinical and multiresolution features for glaucoma detection and its classification using fundus images. Biocybernetics and Biomedical Engineering, v.38, p.329-341,2018.

[11] Mantravadi A. V., Vahhar, N. (2015). Glaucoma. Elsevier,Volume 42, Issue 3, 2015, Pages 437-449, ISSN: 0095-4543.

[12] Robin, X., Turck, N., Hainard, A., Tiberti, N., Lisacek, F., Sanchez, J. C., \& Müller, M. (2015). pROC: display and analyze ROC curves. R Package Version, 1 .

[13] Samanta, S., Ahmed, S. S., Salem, M. A. M. M., Nath, S. S., Dey, N., \& Chowdhury, S. S. (2015). Haralick features based automated glaucoma classification using back propagation neural network. In Proceedings of the 3rd International Conference on Frontiers of Intelligent Computing: Theory and Applications (FICTA) 2014 (pp. 351-358). Springer, Cham.

[14] Septiarini, A., Khairina, D. M., Kridalaksana, A. H., \& Hamdani, H. (2018). Automatic glaucoma detection method applying a statistical approach to fundus images. Healthcare informatics research, 24(1), 53-60.

[15] Tham, Y. C., Li, X., Wong, T. Y., Quigley, H. A., Aung, T., \& Cheng, C. Y. (2014). Global prevalence of glaucoma and projections of glaucoma burden through 2040: a systematic review and meta-analysis. Ophthalmology, 121(11), 2081-2090.

[16] Zahoor, M. N., \& Fraz, M. M. (2018). A Correction to the Article "Fast Optic Disc Segmentation in Retina Using Polar Transform". IEEE Access, 6, 4845-4849. 